\section{FORTRAN IV programs for fuzzy set analysis}

\author{
MICHAEL SMITHSON \\ James Cook University, Queensland, Australia
}

This paper describes a series of programs, written in FORTRAN IV for the DEC 1091 computer, that performs various kinds of data analysis using fuzzy set concepts and techniques. These programs are based on standard FORTRAN 77 and call no special subroutines, and thus are highly portable. Since fuzzy set theory and its applications still are rather unfamiliar to most behavioral and social scientists, this paper begins with a brief review of concepts, followed by descriptions of the models and statistics used in these programs. The subsequent section describes the input and output of the programs themselves. Finally, program limitations and system requirements are discussed.

Fuzzy Set Concepts and Techniques. A set is fuzzy when an element may belong partially (or in degree) to it, rather than belonging either totally or not at all. Formally, fuzzy sets are those in which the characteristic function is allowed to take values in the $[0,1]$ interval instead of the classic restriction to the pair of values $\{0,1\}$. Zadeh (1965) proposed the first complete fuzzy set theory, which since has seen many modifications and refinements. The programs described in this paper, however, are based on foundations in fuzzy set theory that have rather wide acceptance among both theorists and researchers.

Given data points $\mathrm{X}_{\mathbf{i}}$, the customary notation is to denote membership values in a fuzzy set by $\mu_{\mathrm{i}}$, which formally distinguishes between elements and their respective degrees of membership in the referent set. In the discussion to follow, measures and operators will be described for the fuzziness of a set, intersection between two fuzzy sets, and fuzzy set inclusion.

Since one of the appeals of fuzzy set theory is its conceptual basis for categories with blurred edges, it seems crucial to have an appropriate measure of how fuzzy a category actually is. Measures of fuzziness have been much discussed in the fuzzy set literature (see De Luca \& Termini, 1972; Knopfmacher, 1975; Loo, 1977). The approach used in the programs described here follows Smithson's (1982) generalization of relative variation. The specific measure used is

$$
\mathrm{T}_{2}=1-\left[\left(\sum_{\mathrm{i}=1}^{\mathrm{N}} \mu_{\mathrm{i}}^{2}-\mathrm{N} \bar{\mu}^{2}\right) /\left(\sum_{\mathrm{i}=1}^{\mathrm{N}} \mathrm{Q}_{\mathrm{i}}^{2}-\mathrm{N} \overline{\mathrm{Q}}^{2}\right)\right]^{1 / 2},
$$

where the $Q_{i}$ equal 0 or 1 on the following basis: (1) The number of 1-valued $Q_{i}$ equals the whole-number part of

The author is affiliated with the Behavioural Sciences Department, James Cook University, Queensland 4811, Australia.
$N \bar{\mu}$. (2) The remaining fractional part of $N \bar{\mu}$ is represented by a 0 -valued $\mathrm{Q}_{\mathrm{i}}$ if it is less than 0.5 ; otherwise, it is represented by a 1 -valued $Q_{i}$. (3) All remaining $Q_{i}$ equal 0.

$1-T_{2}$ is the proportion of maximum possible variation in the $\mu_{\mathrm{i}}$ given $\mathrm{N}$ and $\bar{\mu}$, so $\mathrm{T}_{2}$ becomes 0 when all $\mu_{\mathrm{i}}$ take on only values of 0 and 1 , and takes the value of 1 when all $\mu_{\mathrm{i}}$ are equal (which is the condition in which the set is so fuzzy that it fails to distinguish among any gradations of membership). One of the advantages of $T_{2}$ is that it can be partitioned by individual subject, stimulus, or referent set along the lines suggested in standard ANOVA procedures (further details are available in the relevant program documentation and also in Smithson, 1982).

Fuzzy set intersection and union are defined in a manner that reduces to ordinary set union and intersection, and therefore incorporates familiar properties. Likewise, as in classical set theory and logic, intersection is identified with logical "and" while union is identified with logical conjunctive "or." It is also possible to define the disjunctive "or" for fuzzy sets. Several different operators have been proposed for intersection and union in fuzzy set theory (for reviews, see Smithson, 1984, and Trillas, Alsina, \& Valverde, 1982). The most widely accepted operators are the min-max family, first proposed by Zadeh (1965). The product operators are probably the second most popular. The list given below contains all the operators included in the program that performs multivariate analysis using "and" and "or" models. In these formulas, $\mathrm{A}\left(\mu_{1}, \mu_{2}\right)$ denotes "and" (or intersection), $\mathrm{O}\left(\mu_{1}, \mu_{2}\right)$ denotes the conjunctive "or" (union), and $\mathrm{XO}\left(\mu_{1}, \mu_{2}\right)$ denotes the disjunctive "or."

Min-max operators are:

$\mathrm{A}\left(\mu_{1}, \mu_{2}\right)=\min \left(\mu_{1}, \mu_{2}\right)$,

$\mathrm{O}\left(\mu_{1}, \mu_{2}\right)=\max \left(\mu_{1}, \mu_{2}\right)$, and

$\mathrm{XO}\left(\mu_{1}, \mu_{2}\right)=\min \left[\max \left(\mu_{1}, \mu_{2}\right), 1-\min \left(\mu_{1}, \mu_{2}\right)\right]$.

Product operators:

$\mathrm{A}\left(\mu_{1}, \mu_{2}\right)=\mu_{1} \mu_{2}$,

$\mathrm{O}\left(\mu_{1}, \mu_{2}\right)=\mu_{1}+\mu_{2}-\mu_{1} \mu_{2}$, and

$\mathrm{XO}\left(\mu_{1}, \mu_{2}\right)=\left(1-\mu_{1} \mu_{2}\right)\left(\mu_{1}+\mu_{2}-\mu_{1} \mu_{2}\right)$.

Bounded sum operators:

$\mathrm{A}\left(\mu_{1}, \mu_{2}\right)=\max \left(0, \mu_{1}+\mu_{2}-1\right)$,

$\mathrm{O}\left(\mu_{1}, \mu_{2}\right)=\min \left(1, \mu_{1}+\mu_{2}\right)$, and

$\mathrm{XO}\left(\mu_{1}, \mu_{2}\right)=1-\left|1-\left(\mu_{1}+\mu_{2}\right)\right|$.

q-type operators:

$\mathrm{A}\left(\mu_{1}, \mu_{2}\right)=\min \left(\mu_{1}, \mu_{2}\right)-\mathrm{qZ}$,

$\mathrm{O}\left(\mu_{1}, \mu_{2}\right)=\max \left(\mu_{1}, \mu_{2}\right)+\mathrm{qZ}$, and

$\mathrm{XO}\left(\mu_{1}, \mu_{2}\right)=\mathrm{H}+\mathrm{q}^{2} \mathrm{Z}$,

where $Z=.5\left(1-\left|\mu_{1}-\mu_{2}\right|-\left|1-\left(\mu_{1}+\mu_{2}\right)\right|\right) \quad$ and $\mathrm{H}=.5\left(1+\left|\mu_{1}-\mu_{2}\right|-\left|1-\left(\mu_{1}+\mu_{2}\right)\right|\right)$, for $\mathrm{q} \geq 0$ and $\leq 1$. 
The q-type family incorporates the min-max and bounded sum operators as special cases (when $q=0$ and $q=1$, respectively).

A concept closely related to set intersection is fuzzy set overlap. Overlap concerns the relative amounts of membership shared by two sets. Following suggestions from Bezdek (1974), Smithson (1982) developed a measure of overlap that used the product operator for intersection:

$\mathrm{M}_{\mathrm{jk}}=\sum_{\mathrm{i}=1}^{\mathrm{N}} \mu_{\mathrm{ij}} \mu_{\mathrm{ik}} /\left(\sum_{\mathrm{i}=1}^{\mathrm{N}} \mu_{\mathrm{ij}}^{2} \sum_{\mathrm{i}=1}^{\mathrm{N}} \mu_{\mathrm{ik}}^{2}\right)^{1 / 2}$

Fuzzy set inclusion also is defined in terms similar to those for classical set theory. Fuzzy set $j$ is said to include fuzzy set $\mathbf{k}$ if each element is at least as strong a member of $\mathbf{j}$ as it is of $\mathbf{k}$. In membership functional terms, $\mu_{\mathrm{ij}} \geq \mu_{\mathrm{ik}}$. Several measures of inclusiveness have been proposed, but the one used in this program series is from Smithson (1982):

$\mathrm{I}_{\mathbf{k j}}=\sum_{\mathrm{i}=1}^{\mathrm{N}} \max \left(\mathrm{O}, \mu_{\mathrm{ij}}-\mu_{\mathrm{ik}}\right) / \sum_{\mathrm{i}=1}^{\mathrm{N}}\left|\mu_{\mathrm{ij}}-\mu_{\mathrm{ik}}\right|$.

$I_{k j}$ measures the extent to which set $k$ is a subset of $j$, by computing the proportion of deviations from the diagonal that lie above it. $I_{k j}=1$ when set $j$ entirely includes set $j$, and $\mathrm{I}_{\mathbf{k j}}=1-\mathrm{I}_{\mathbf{j k}}$.

Virtually all of the concepts and statistics introduced thus far have been applied in psychological and social psychological research. Measures of fuzziness have been used to assess the definiteness of diagnostic categories for mental retardation (Weber, 1981) and also for delineating fuzziness of common words for helping behavior in semantic space (Smithson, Amato, \& Pearce, 1983, chap. 5). They have also been used in measurement problems. Grichting (1984) applied them to measuring boundary permeability in organizational structures, and Smithson, Amato, and Pearce (1983, chap. 4) used them to determine whether subjects were using bipolar scales as continua or as dichotomies.

Overlap coefficients have also found several applications. Pearce (1983) used them for input to a standard hierarchical clustering routine in his inquiry concerning the fuzzy overlap of tourist roles. Smithson et al. (1983, chap. 5) applied both overlap coefficients and measures of inclusion to developing a hierarchical taxonomy of some $\mathbf{4 0}$ common terms for helping behavior. Hierarchy in this work actually referred to the degree of inclusivity of various terms as lexical fuzzy sets. Details of this application also are available in Smithson (1982).

Applications for fuzzy conjunctions ("and," "or," etc.) are still rare. Some cognitive psychologists have explored them in an attempt to assess their capacity to model the ways in which people actually aggregate fuzzy sets (cf. Oden, 1977; Zimmermann \& Zysno, 1980). However, least-squares methods for fitting such connectives to data have only recently been developed (Smithson, 1984). These methods have been successfully applied to ANOVA and regression-type problems. The models in the relevant program in this series use a generalization of the fuzzy connective, which has two forms:

$\mu_{1} \& \mu_{2}=\mathrm{FO}\left(\mu_{1}, \mu_{2}\right)+(1-\mathrm{F}) \mathrm{A}\left(\mu_{1}, \mu_{2}\right)$.

$\mu_{1} @ \mu_{2}=\mathrm{FO}\left(\mu_{1}, \mu_{2}\right)+(1-\mathrm{F}) \mathrm{XO}\left(\mu_{1}, \mu_{2}\right)$,

where $\mathrm{O} \leq \mathrm{F} \leq 1$. Formula 8 was motivated by the observation that for a large class of $\mathrm{A}$ and $\mathrm{O}$ connectives, unweighted addition [i.e., $.5\left(\mu_{1}+\mu_{2}\right)$ ] lies exactly halfway between fuzzy "and" and fuzzy conjunctive "or." Thus, "and" and "or" both are equal and opposite deviations from linearity, a fact that renders them useful for modeling certain kinds of interaction models (see Smithson, 1984, for further explanations and results). The @ connective was motivated by a need to be able to model "or" connectives of varying degrees of exclusivity.

Both Formulas 8 and 9 suggest least-squares solutions for the F parameter, and, under the proviso that only the dependent variable is permitted a linear transformation, solutions are obtainable. Under the same condition, leastsquares solutions can also be obtained for the q parameter in the q-type operator family. This result is useful when the investigator wishes to fit a pure $\mathrm{A}, \mathrm{O}$, or $\mathrm{XO}$ connective to data but wants to find an optimal connective between the min-max and bounded sum families. The general model is

$K Y_{i}^{\prime}+C=A_{i}+P D_{i}$,

where $Y_{i}^{\prime}$ is the dependent variable, and $\mathrm{K}$ and $\mathrm{C}$ are the constants for the appropriate linear transformation (they are solved simultaneously with $\mathrm{P}$ ). The nature of $\mathrm{A}, \mathrm{P}$, and $D$ depends on the specific model chosen for analysis, and they are provided below:

$\&$ connective:

$\mathrm{A}_{\mathrm{i}}=\mathrm{A}\left(\mu_{1 \mathrm{i}}, \mu_{2 \mathrm{i}}\right)$,

$\mathrm{D}_{\mathrm{i}}=\mathrm{O}\left(\mu_{1 \mathrm{i}}, \mu_{2 \mathrm{i}}\right)-\mathrm{A}_{\mathrm{i}}$, and

$\mathrm{P}=\mathrm{F}$.

@ connective:

$\mathrm{A}_{\mathrm{i}}=\mathrm{XO}\left(\mu_{1 \mathrm{i}}, \mu_{2 \mathrm{i}}\right)$,

$\mathrm{D}_{\mathrm{i}}=\mathrm{O}\left(\mu_{1 \mathrm{i}}, \mu_{2 \mathrm{i}}\right)-\mathrm{A}_{\mathrm{i}}$, and

$\mathrm{P}=\mathrm{F}$.

q-type operator for "and":

$A_{\mathrm{i}}=\min \left(\mu_{1 \mathrm{i}}, \mu_{2 \mathrm{i}}\right)$,

$\mathrm{D}_{\mathrm{i}}=\mathrm{Z}_{\mathrm{i}}$, and

$\mathbf{P}=-\mathbf{q}$. 
Q-type operator for conjunctive "or":

$\mathrm{A}_{\mathrm{i}}=\max \left(\mu_{1 \mathrm{i}}, \mu_{2 \mathrm{i}}\right)$,

$\mathrm{D}_{\mathrm{i}}=\mathrm{Z}_{\mathrm{i}}$, and

$\mathrm{P}=\mathrm{q}$.

q-type operator for disjunctive "or":

$\mathrm{A}_{\mathrm{i}}=\mathrm{H}_{\mathrm{i}}$,

$\mathrm{D}_{\mathrm{i}}=\mathrm{Z}_{\mathrm{i}}$, and

$\mathrm{P}=\mathrm{q}^{2}$.

The program described in this series performs all of the analyses listed above, and in the \& or @ cases, does so for any of the connectives defined in Formulas 2-5. A larger program is being developed to handle multipleconnective, multivariate models. Multiple-connective models are not as straightforward as the three-variable, single-connective models described here (see Smithson, 1984, for discussions of multiple-connective models).

Input Requirements and Limitations. The first four programs in the series (FUZZY1-FUZZY4) read a threeway matrix of data on channel 7 in which the stimuli are indexed by rows, categories (or referent sets) by columns, and subjects by submatrices stacked vertically in the data file. The data themselves consist of subjects' ratings of the degree of membership of each stimulus in each category. These ratings may be entered in any format, but they must of course fall within the $[0,1]$ interval. The program parameters that control various options or inform the program of the matrix size are contained in a single row on top of the data matrix itself. These programs are limited to 25 stimuli, 25 categories, and 100 subjects, although it is not difficult to alter these limitations. Each of them occupies $4 \mathrm{~K}$ to $6 \mathrm{~K}$ of memory.

The fifth program, ANDOR, performs least squares for the three-variable, single-connective models described above, and it expects a matrix with three columns. The first column corresponds to the dependent variable, and the second two to the independent variables. It also requires that the data reside on channel 7 , and assumes that the data consist of membership ratings in the $[0,1]$ interval. The first two rows of the input file are reserved for parameters that control program options and inform the program of the matrix size. The greatest number of subjects permitted is 500 , and the program itself occupies about $12 \mathrm{~K}$ of memory.

Program Options, Features, and Output. FUZZY1 and FUZZY2 both analyze fuzziness, using the measure of fuzziness defined in Formula 1. FUZZY1 performs this analysis for subjects and stimuli. Options allow for fuzziness indexes to be computed on all stimuli for each subject, on all stimuli averaged over all subjects, or on the subjects themselves. FUZZY2 computes fuzziness measures for categories or sets. Again, program options allow these indexes to be computed for individual subjects or over all subjects. The program also provides partitioned indexes of relative variation due to fuzziness, on the one hand, and interindividual differences, on the other. Both programs output on channel 6.

FUZZY3 computes overlap coefficients using Formula 6 . These coefficients are output in a lower half triangular matrix similar in appearance to an ordinary correlation matrix. A program option allows for these coefficients to be computed for each subject or overall. FUZZY4 computes inclusion coefficients as defined in Formula 7. Again, an option permits the coefficients to be computed for either individual subjects or overall. The results are output in a full matrix. An additional option allows the program to produce a hierarchical ordering of the categories, on the basis of their respective inclusion relationships. These two programs also output on channel 6.

ANDOR performs least-squares analysis for the threevariable, single-connective models discussed above (Formulas 10-15). The main options in the program permit the user to select whether they are going to test a $\&$ model or a @ model, or compute optimal q values for a pure "and," conjunctive "or, " or disjunctive "or." If users choose either \& or @, then they may also select the type of connective they wish (Formulas 2-5). An additional set of options allows exponential and/or multiplicative weights for the independent variables. The computer searches for optimal weights within a range specified by the user, and uses stopping rules based on a criterion for improvement in $\mathrm{R}^{2}$ or a maximum number of iterations, both of which also are supplied by the user. ANDOR outputs on channel 6 , and produces the values for $\mathrm{K}, \mathrm{C}, \mathrm{P}$, $R^{2}$ and the weight values on the iteration to which the output refers.

Availability. The programs have their own selfcontained documentation, and the listings themselves are liberally sprinkled with comments to aid the experienced user who wishes to make modifications. The entire series, or any of the programs in it, are available free of charge from the following postal address: Michael Smithson, Behavioural Sciences Department, James Cook University, Queensland 4811, Australia.

\section{REFERENCES}

Bezdek, I. C. (1974). Numerical taxonomy with fuzzy sets. Journal of Mathematical Biology, 1, 57-71.

De LuCA, A., \& TerminI, S. (1972). A definition of nonprobabilistic entropy in the setting of fuzzy sets theory. Information and Control, 20, 301-312.

Grichting, W. L. (1984). Security vs. liberty. Lanham, MD: American Universities Press.

KNOPFMACHER, K. (1975). On measures of fuzziness. Journal of Mathematical Analysis and Applications, 49, 529-534.

Loo, S. G. (1977). Measures of fuzziness. Cybernetica, 20, 201-207.

ODEN, G. C. (1977). Integration of fuzzy logical information. Journal of Experimental Psychology, 106, 565-575.

Pearce, P. L. (1983). The social psychology of tourist behaviour. Oxford, England: Pergamon Press.

SMITHSON, M. (1982). Applications of fuzzy set concepts to behavioral sciences. Journal of Mathematical Social Sciences, 2, 257-274. 
SMithson, M. (1984). Multivariate analysis using 'and' and 'or.' Journal of Mathematical Social Sciences, 7, 231-251.

Smithson, M., Amato, P. R., \& Pearce, P. L. (1983). Dimensions of helping behaviour. Oxford, England: Pergamon Press.

Trillas, E., Alsina, C., \& Valverde, L. (1982). Do we need max, min, and $1-\mathrm{j}$ in fuzzy set theory? In R. R. Yager (Ed.), Fuzzy set and possibility theory: Recent advances. Oxford, England: Pergamon Press.
WEBER, L. P. (1981). Labels of retardation. Unpublished honors thesis, James Cook University.

ZADEH, L. A. (1965). Fuzzy sets. Information and Control, 8, 338-353.

ZIMMERMAN, H.-J., \& ZYSNO, P. (1980). Latent connectives in human decision making. Fuzzy Sets and Systems, 4, 37-51.

(Revision accepted for publication September 25, 1984.) 